INNOVATIONS IN PRIMARY CARE

\title{
Empowering Community Health Workers With Mobile Technology to Treat Diabetes
}

\author{
Sean Duffy, $M D^{1}$ \\ Jim Svenson, MD, MS \\ Mark Kelly ${ }^{1}$ \\ Alejandro Chavez ${ }^{2}$ \\ Ann Fam Med 2019;17:176. https://doi.org/10.1370/afm.2361.
}

\section{THE INNOVATION}

We designed and implemented a clinical decision support application (app) for smartphones to enable lay community health workers (CHWs) to manage diabetes in rural Guatemala.

The global prevalence of diabetes has doubled since 1980 and low- and middle-income countries (LMICs) - such as Guatemala-are bearing the brunt of this epidemic., These countries are ill-equipped to deal with the rise in diabetes and other chronic diseases due to inadequate health system infrastructure and critical workforce shortfalls. ${ }^{3,4}$ Health systems in LMICs are increasingly utilizing CHWs and mobile technology to fill gaps in chronic disease care and there is mounting evidence that these interventions can improve outcomes for patients with diabetes and other chronic diseases. ${ }^{5-17}$ In these programs, CHWs most often serve a support role and mobile health apps are generally used for data collection, communication, and patient education rather than to direct treatment. Our program is unique among published diabetes interventions in the use of a smartphone app to provide algorithmic clinical decision support to CHWS, enabling them to directly deliver diabetes care, including the titration of oral medications and management of complications.

\section{WHO \& WHERE}

The Friends of San Lucas (FOSL) is a nonprofit organization providing health care and social services to a predominantly indigenous population in the town of San Lucas Tolimán and the surrounding rural villages in southwestern Guatemala. FOSL partners with local CHW/s to serve patients in these rural villages. The University of Wisconsin and Stanford University have long-standing collaborations with FOSL.

Conflicts of interest: authors report none.

\section{Corresponding author}

Sean Duffy, MD

University of Wisconsin School of Medicine and Public Health

Department of Family Medicine and Community Health

1100 Delaplaine Court

Madison, WI 53715

sean.duffy@fammed.wisc.edu

\section{HOW}

Working with local medical providers, we developed clinical protocols for diabetes care based on international guidelines. We integrated these protocols into a clinical decision support smartphone app utilizing CommCare, a mobile technology platform commonly used by health programs in the developing world.

We trained CHWs working under the supervision of local physicians (1) to use the app and (2) to provide basic diabetes care. The CHWs recruited patients from their communities, who provided their informed consent to participate in the program. They meet with these patients monthly and, with the assistance of the app, assess diabetes control, effectiveness and tolerability of treatment, presence of complications, and diabetes self-care. Using information entered by the CHWs, including point-of-care glucose and hemoglobin $A_{1 c}$ testing, the app then provides the CHWs with recommendations regarding titration of metformin and/or glyburide, management of diabetes complications, lifestyle counseling, and referral to a physician as needed.

After each visit, the CHWs upload data from the app to a secure server, where it is then reviewed by a physician, who can make further recommendations and adjustments to the treatment plan as needed.

\section{LEARNING}

The smartphone app has proven to be safe, feasible, and satisfying to patients and CHWs, and early data suggests efficacy in improving diabetes care. Compared with baseline values, mean $A_{1 c}$ has improved at each time point, decreasing by $1.5 \%$ at 3 months ( $n=46, P=0.01$ ) and $1.6 \%$ at 6 months ( $n=21$, $P=0.02$ ); this trend appears to continue through 12 months. The proportion of patients meeting treatment goals for $A_{1 c}$ increased from $13.0 \%$ to $34.8 \%(P=0.09)$ of patients followed to 3 months. Data collection through 1 year is ongoing.

If results continue to be positive, this app may find utility in other low-resource settings. We plan to freely share the app through the CommCare platform. Our model for diabetes care-utilizing CHWs empowered by smartphone technologycould potentially be applied to other chronic health conditions, including hypertension and heart failure.

Author affiliations, references, and acknowledgments available at http://www.annfammed.org/content/17/2/176/suppl/DC1.

Key words: global health; diabetes mellitus; community health workers; mobile health; decision support systems, clinical; Guatemala 